\title{
Analysis of human emboli and thrombectomy forces in large-vessel occlusion stroke
}

\author{
Yang Liu, PhD, ${ }^{1}$ Yihao Zheng, PhD, ${ }^{2}$ Adithya S. Reddy, ${ }^{3}$ Daniel Gebrezgiabhier, ${ }^{3}$ Evan Davis, ${ }^{1}$ \\ Joshua Cockrum, ${ }^{3}$ Joseph J. Gemmete, MD, ${ }^{3,4}$ Neeraj Chaudhary, MD, ${ }^{3,4}$ Julius M. Griauzde, MD, ${ }^{4}$ \\ Aditya S. Pandey, MD, ${ }^{3}$ Albert J. Shih, PhD, ${ }^{1}$ and Luis E. Savastano, MD, PhD ${ }^{3,5}$ \\ 'Department of Mechanical Engineering, University of Michigan, Ann Arbor, Michigan; 2Department of Mechanical Engineering, \\ Worcester Polytechnic Institute, Worcester, Massachusetts; Departments of ${ }^{3}$ Neurosurgery and ${ }^{4}$ Radiology, University of \\ Michigan, Ann Arbor, Michigan; and ${ }^{5}$ Department of Neurosurgery, Mayo Clinic, Rochester, Minnesota
}

\begin{abstract}
OBJECTIVE This study's purpose was to improve understanding of the forces driving the complex mechanical interaction between embolic material and current stroke thrombectomy devices by analyzing the histological composition and strength of emboli retrieved from patients and by evaluating the mechanical forces necessary for retrieval of such emboli in a middle cerebral artery (MCA) bifurcation model.
\end{abstract}

METHODS Embolus analogs (EAs) were generated and embolized under physiological pressure and flow conditions in a glass tube model of the MCA. The forces involved in EA removal using conventional endovascular techniques were described, analyzed, and categorized. Then, 16 embolic specimens were retrieved from 11 stroke patients with largevessel occlusions, and the tensile strength and response to stress were measured with a quasi-static uniaxial tensile test using a custom-made platform. Embolus compositions were analyzed and quantified by histology.

RESULTS Uniaxial tension on the EAs led to deformation, elongation, thinning, fracture, and embolization. Uniaxial tensile testing of patients' emboli revealed similar soft-material behavior, including elongation under tension and differential fracture patterns. At the final fracture of the embolus (or dissociation), the amount of elongation, quantified as strain, ranged from 1.05 to $4.89(2.41 \pm 1.04$ [mean \pm SD]) and the embolus-generated force, quantified as stress, ranged from 63 to $2396 \mathrm{kPa}(569 \pm 695 \mathrm{kPa})$. The ultimate tensile strain of the emboli increased with a higher platelet percentage, and the ultimate tensile stress increased with a higher fibrin percentage and decreased with a higher red blood cell percentage.

CONCLUSIONS Current thrombectomy devices remove emboli mostly by applying linear tensile forces, under which emboli elongate until dissociation. Embolus resistance to dissociation is determined by embolus strength, which significantly correlates with composition and varies within and among patients and within the same thrombus. The dynamic intravascular weakening of emboli during removal may lead to iatrogenic embolization.

https://thejns.org/doi/abs/10.3171/2019.12.JNS192187

KEYWORDS stroke; large-vessel occlusion; embolus; thrombectomy; hemodynamic forces; vascular disorders

$\mathrm{M}$ ECHANICAL thrombectomy using catheter and stent technology has proven to be safe and efficacious in improving clinical outcomes in patients with stroke due to large-vessel occlusion (LVO). ${ }^{19}$ Successful recanalization, or modified Treatment in Cerebral Infarction scores of $2 b$ and $3,{ }^{3}$ is routinely achieved in $80 \%-90 \%$ of cases with currently available stent retriever and aspiration thrombectomy technologies and is associated with good clinical outcomes. However, complete recanalization (a modified Treatment in Cerebral Infarction score of 3), which is known to be associated with the best neurological outcome, is achieved in $<50 \%$ of patients. ${ }^{10}$ In addition, with current devices, iatrogenic embolization can cause macro- and microvascular occlusions, ${ }^{11}$ and multiple passes may be necessary to recanalize LVOs, thus necessitating longer procedure time and risking further neuronal injury. ${ }^{23}$

Given the limitations of current thrombectomy devices, we sought to gain an understanding of the forces driving the complex interaction between emboli and thrombec-

ABBREVIATIONS ADAPT $=$ a direct aspiration first-pass technique; CAPTIVE $=$ continuous aspiration prior to intracranial vascular embolectomy; $\mathrm{EA}=$ embolus analog; LVO = large-vessel occlusion; MCA = middle cerebral artery; $\mathrm{RBC}=$ red blood cell; $\varepsilon_{\mathrm{ut}}=$ ultimate tensile strain; $\sigma_{\mathrm{ut}}=$ ultimate tensile stress.

SUBMITTED August 15, 2019. ACCEPTED December 2, 2019.

INCLUDE WHEN CITING Published online February 28, 2020; DOI: 10.3171/2019.12.JNS192187. 
tomy devices by analyzing retrieval in a middle cerebral artery (MCA) bifurcation model and evaluating the mechanical forces and histological composition of emboli obtained from patients with LVO stroke. In addition, by correlating histological composition with tensile properties of patients' emboli, we lay the groundwork to generate representative embolus analogs (EAs) for evaluation of future thrombectomy devices.

\section{Methods}

First, to study the thrombectomy procedure, an anatomical model of an LVO involving the MCA bifurcation with physiological flow and pressures was created. Video recording of the thrombectomy procedure using a direct aspiration first-pass technique (ADAPT) and continuous aspiration prior to intracranial vascular embolectomy (CAPTIVE) technique (described below) was performed. The EAs were subjected to tensional forces leading to elongation and fracture during material removal. In addition, a destructive tensile test of patient-derived emboli was used to analyze the properties of elongation and fracture. Then, histological analysis of the emboli with structural quantification was performed.

\section{Mechanical Thrombectomy Model}

A glass model (Farlow's Scientific Glassblowing, Inc.) of the intracranial internal carotid artery and the anterior cerebral artery and MCA was used to visualize the retrieval process. The model was connected to a hydraulic system that consisted of a peristaltic pump, tubing, and variable resistance escape valves.

EAs were generated by mixing plasma and red blood cells (RBCs) from human blood with a volume ratio of 9:1 . A calcium chloride solution (2.3 wt $\%$ ) was then added to the mixture with a volume ratio of 1:10 to induce coagulation. ${ }^{6,14}$ The mixture was allowed to rest at $37^{\circ} \mathrm{C}$ for 90 minutes in a polyvinyl chloride tube with a diameter of $15 \mathrm{~mm}$. Then the EAs were removed from the polyvinyl chloride tubes, sectioned into 5-mm-long fragments, and injected into the tubing system through an injection port (with a 5-mm diameter to prevent any tissue deformation), and they were carried distally by physiological flow into the MCA bifurcation.

Mechanical thrombectomy of the EAs was conducted by the following: 1) ADAPT employing an aspiration catheter (ACE 68; Penumbra) connected to a full vacuum (-650 mm Hg vacuum, pump model 01-12-405; Allied Healthcare) (Fig. 1A);20 and 2) CAPTIVE with Solitaire Platinum revascularization device (Medtronic) and an aspiration catheter (ACE 68) (Fig. 1B). ${ }^{1}$ The thrombectomy procedures were performed 10 times with each technique, and videos were recorded for analysis by a team of mechanical engineers and neurointerventionalists, including radiologists and neurosurgeons. The forces involved in the removal process were analyzed, described, and categorized. The material responses of the EAs to the action of the thrombectomy devices were recorded and analyzed. The number of passes needed to recanalize the obstructed artery was quantified. After each pass of mechanical thrombectomy, the emboli were collected in a tank and photographed next to a ruler. The emboli area was calculated using Fiji v.2.0.0 software. Emboli smaller than 1 $\mathrm{mm}$ were excluded from analysis.

\section{Patient Embolus Collection}

Collection and handling of specimens and data were performed following the protocol approved by the institutional review board. Emboli were prospectively obtained from patients who underwent thrombectomy by the combination of a stent retriever (Solitaire Platinum) and an aspiration catheter (ACE 68) using the CAPTIVE technique (Fig. 2A insert). ${ }^{16}$ Seventy-five thrombectomy procedures for LVO were included in this study. Thirty-seven emboli removed from 20 patients were of sufficient size (at least $10 \mathrm{~mm}$ in length and $3 \mathrm{~mm}$ in diameter) for tensile testing. The remaining emboli were too small for testing, or the embolus material could not be located despite meticulous inspection of the devices.

\section{Tensile Test Setup}

The tensile test was carried out using a customized tensile test machine (Fig. 2A) that consisted of a computer-controlled linear stage (200cri; Siskiyou) with a force sensor (Gamma; ATI Industrial Automation) to measure the tensile force $(F)$. The embolus was gripped by two hemostats, one fixed to the linear stage and the other to the stationary force sensor. The embolus was pulled at a rate of $0.2 \mathrm{~mm} / \mathrm{sec}$ to negate the viscoelastic effect until fracturing and the tensile force was measured at $50 \mathrm{~Hz}$. Two video cameras ( $\alpha 6000$; Sony) recorded digital images of the deformed embolus from the top and side at 30 frames per second. Simultaneous images from the two cameras were used to estimate the cross-sectional area of embolus during tensile testing. Two fiducials were added on both ends of the embolus before pulling to track the elongation.

To quantify the emboli tensile properties, true stress $(\sigma)$ and engineering strain $(\varepsilon)$ were calculated and stressstrain curves were generated for each sample (Fig. 2). The true stress is the loading on a material per unit area caused by force (i.e., aspiration catheter- and stent retriever-related pulling and, in our case, the hemostats' pull), which then causes the material to deform (in our case, to elongate until the embolus fractures). The engineering strain quantifies the elongation of the embolus. The method used to calculate true stress and engineering strain is described in the Supplemental Material. At fracture, the embolus has an ultimate tensile stress $\left(\sigma_{\mathrm{ut}}\right)$ and an ultimate tensile strain $\left(\varepsilon_{\mathrm{ut}}\right)$.

\section{Quantification of Embolus Composition}

After tensile testing, the emboli were fixed in 10\% neutral buffered formalin solution, embedded in paraffin, and sliced using a microtome at 5- $\mu \mathrm{m}$ slice thickness. Slides were stained with $\mathrm{H} \& \mathrm{E}$ to highlight the RBCs from the fibrin matrix, and immunostained with CD61 (anti-ITGB3 antibody [HPA027852; Atlas Antibodies]) for platelet content. ${ }^{9}$ The stained slides were scanned by a microscope (BX51; Olympus) at $\times 40$ magnification, and images were recorded with a digital camera (DP70; Olympus) at 1600 $\times 1200$ resolution. The $\mathrm{H} \& \mathrm{E}$ and CD61 images were pro- 


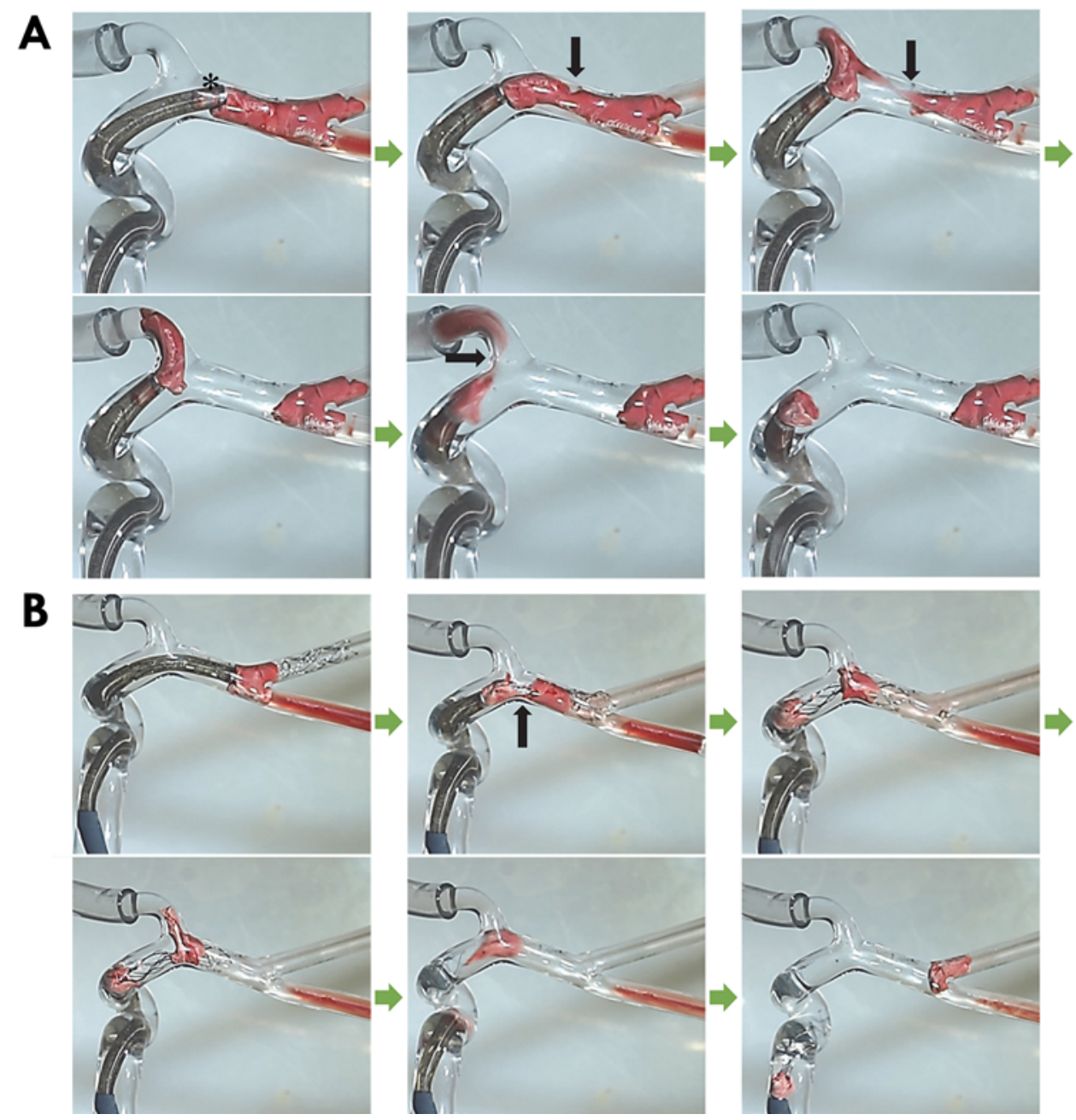

FIG. 1. Sequential images of mechanical thrombectomy in a glass phantom of the internal carotid artery and major intracranial arteries that was pressurized with a saline solution. With EAs embolized into the MCA, and under physiological flow and pressure, thrombectomy was conducted with the ADAPT (A) and the CAPTIVE (B) techniques. After EA engagement, device withdrawal exposed the EAs to tensional forces that led to progressive elongation, thinning, and fracture (black arrows), resulting in partial embolus removal and iatrogenic embolization. The asterisk in A indicates the EA entering the aspiration catheter under a vacuum (engagement segment). Figure is available in color online only.

cessed using ImageJ (v1.52; National Institutes of Health) to count the dark red and dark brown pixels to determine the percentages of RBCs and platelets (Fig. 2C). The rest was assumed to be fibrin.

The tensile stress-strain test and histological results were organized by stroke etiology, as assigned by stroke neurologists blinded to the study results after appropriate workup following standard of care. Specimens were categorized as cardioembolic (assuming a cardiac origin from the heart with structural disease or arrhythmia), atherogenic (ipsilateral carotid artery stenosis $>50 \%$ with no cardiac structural disease or arrhythmia), or cryptogenic emboli (i.e., embolic stroke of unknown etiology; see Table 1 in the Supplemental Material). One specimen originating from a left ventricular assistance device (embolus 8 [Table 1 of the Supplemental Material]) was included in the cardioembolic category.

\section{Results \\ Mechanical Thrombectomy Model}

The forces involved in the removal process employing ADAPT and CAPTIVE techniques (Fig. 1A and 1B, respectively, and Video 1) were analyzed, described, and categorized into retrieval forces, resistance forces, and emboligenic forces (Fig. 3).

VIDEO 1. Mechanical thrombectomy in a glass model of the cerebral arteries using the ADAPT technique and CAPTIVE technique. Copyright University of Michigan. Published with permission. Click here to view.

Retrieval forces favor retrograde removal of emboli and include tensional forces applied by the devices on the emboli (vacuum by aspiration catheters and/or linear pull of stents and catheters) and intravascular pressure distal to the site of occlusion secondary to collateral flow. Re- 

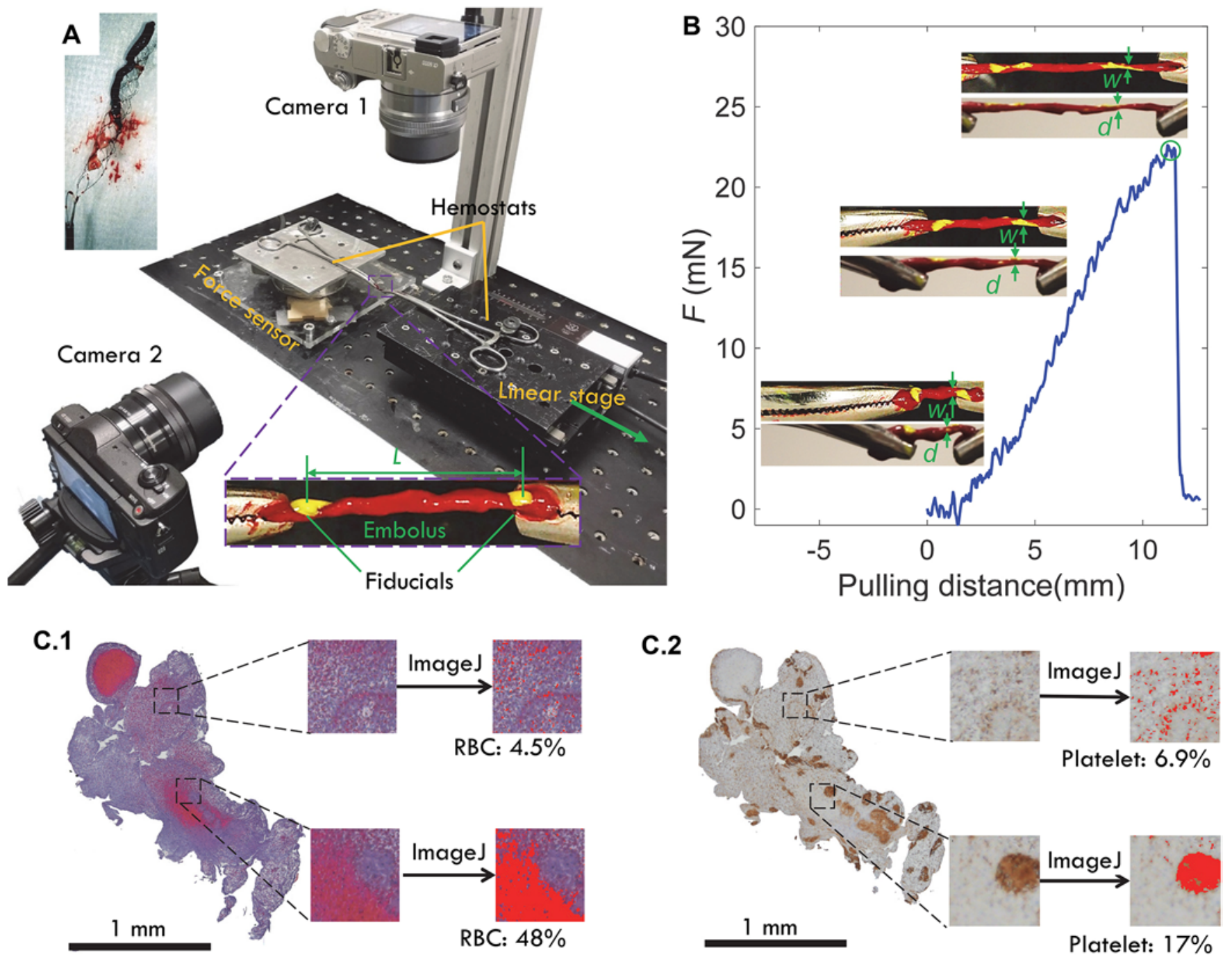

FIG. 2. Tensile testing and quantitative analysis of histological components of emboli. A: Embolic fragments retrieved from patients undergoing mechanical thrombectomy for LVO were harvested (insert) and analyzed in a customized tensile test machine. Emboli were marked with two fiducials and recorded by cameras in anteroposterior and lateral projections and secured by hemostats attached to the linear stage for tensile testing. $L=$ distance between the two fiducials (Supplemental Material). B: Stress-strain curves and videos of the emboli subjected to tension were generated for each sample. In this curve corresponding to embolus $2 \mathrm{~A}$, the pulling force $(F)$ rises as the pulling distance increases. The embolus deforms through elongation and thinning until fracture after $11 \mathrm{~mm}$ of tensile pulling. The widths of the embolus were measured from the camera's top $(w)$ and side $(d)$ images. C: Emboli were processed by histology and $\mathrm{H} \& \mathrm{E}(\mathrm{C} .1)$ and $\mathrm{CD} 61$ immunohistochemistry (C.2) to quantify emboli composition. Figure is available in color online only.

sistance forces refer to the ability of the emboli to resist change and include static friction and adhesion at the embolus-vascular surface interface, the mechanical strength of the embolus opposing the material intruding into branching arteries to deform and enter into the main artery (and into the aspiration catheter if used) for removal, and the arterial pressure proximal to the obstruction (Fig. $3 \mathrm{~A})$. If retrieval forces overcome resistance forces, at least a part of the embolus will become disimpacted, leading to a stage where emboligenic forces favor a downstream migration of embolus fragments under reconstituted flow conditions, including dynamic friction at the embolusvascular surface interface and hemodynamic stress com- prising shear and pressure at the embolus-blood interface (Fig. 3B).

During the ADAPT technique, the aspiration catheters were advanced to the proximal edge of the EAs and connected to the vacuum source for 5 minutes before withdrawal. During this time, EAs were observed to enter the lumen of the aspiration catheter for a few millimeters and then remain in a stable position. The distal region of the aspiration catheter occupied with EAs is the engagement segment (asterisk, Fig. 1A). During aspiration catheter withdrawal, EAs were observed to elongate, decrease in diameter, and undergo multistage fracture that led to embolization into the same vascular territory and other arte- 

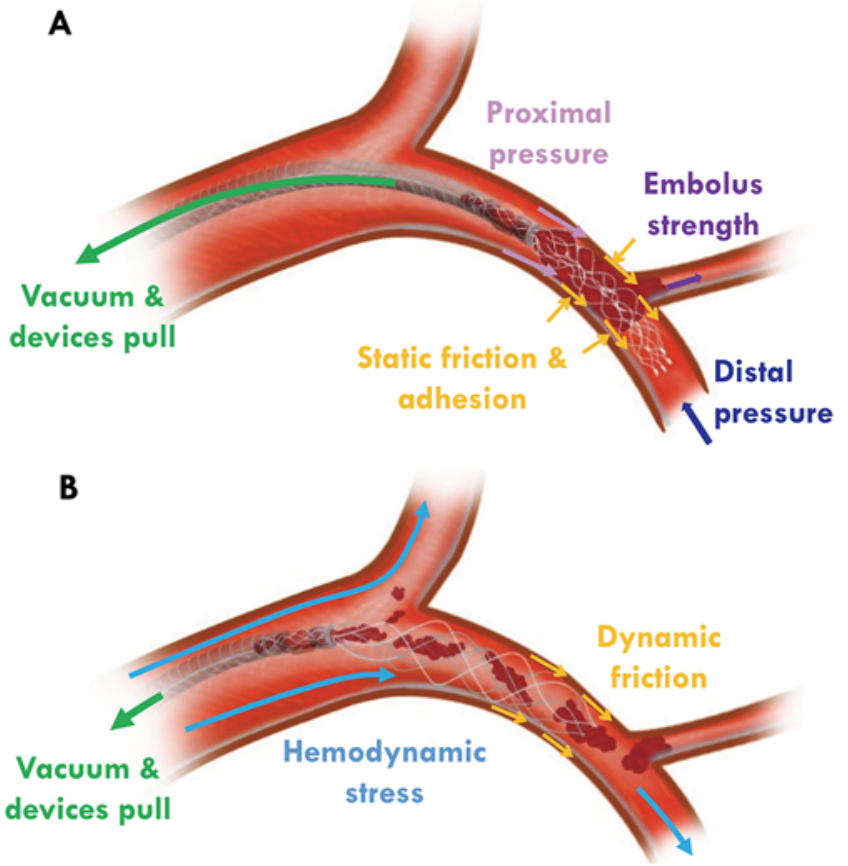

FIG. 3. Interplay of forces involved in the removal of an embolus in LVO with a stent retriever and aspiration catheter during embolus engagement and initial device withdrawal $(\mathbf{A})$ and after embolus disimpaction and removal (B). Figure is available in color online only.

rial branches. In the 10 procedures performed, as shown in Table 1, we observed 1.3 emboli per intervention (SD 0.5), with an average size of $5.95 \mathrm{~mm}$ (SD 6.06). The ADAPT technique required an average of 1.5 attempts (SD 0.5) to recanalize the MCA. For the ADAPT technique, the major retrieval force was the uniaxial tensile force at the engagement segment, leading to EA elongation, thinning, and fracture to overcome the impaction forces at the EA/ phantom interface, mostly consisting of static friction, adhesion, and differential pressure across the EA. After disimpaction of the EA and partial recanalization of the lumen, emboligenic forces included the dynamic friction of the EA at the glass phantom surface and hemodynamic forces (shear and pressure) of the saline solution over the EA surface, causing multiple iatrogenic embolization.

During the CAPTIVE technique, the stent was deployed across the embolus with at least one-third of the stent distal to the embolus and one-third inside the catheter (Fig. 1B). The stent had sufficient radial force to incorporate into the EA and oppose the phantom wall. The aspiration catheter was then advanced. After contacting the EA substance, the catheter was connected to the vacuum source for 5 minutes before simultaneous withdrawal of the stent and catheter. The aspiration catheter and the stent generated uniaxial tensile force that led to stress and strain loading in the EA. During removal, tensional forces dislodged the embolus from the MCA bifurcation, with associated EA elongation, thinning, and fracture (Fig. 1B and Video 1). Stent integration into the EA was weaker at the branching point, with stent deployment confined to the diameter of the distal and smaller lumen, leaving an embol-
TABLE 1. Comparison of ADAPT and CAPTIVE techniques in the glass tube model

\begin{tabular}{lccc}
\hline Technique & No. of Passes & No. of Emboli & Embolus Size $(\mathrm{mm})$ \\
\hline ADAPT & $1.5($ SD 0.5) & $1.3($ SD 0.5) & $5.95($ SD 6.06) \\
\hline CAPTIVE & $1.9($ SD 0.7) & $1.9($ SD 1.8) & $5.88($ SD 6.15) \\
\hline
\end{tabular}

ic substance outside the stent tines. The EA experienced elongation and fracture during device withdrawal and had exposure to hemodynamic forces and friction against the glass phantom, leading to high emboligenic propensity. These mechanisms were consistently associated with an overall decrease in grasp and total retrieval forces concurrent with the increase in proemboligenic forces due to restored flow through the lumen, leading to incomplete embolus removals and iatrogenic embolization. The segment of the EAs that was most consistently removed and was less likely to embolize was at the interface between the aspiration catheter and the stent. In the 10 procedures performed, we observed 1.9 emboli per intervention (SD 1.8), with an average size of $5.88 \mathrm{~mm}$ (SD 6.15). An average of 1.9 passes (SD 0.7) were needed to recanalize the MCA.

\section{Stress and Strain Analysis of Patients' Derived Emboli and Histological Correlation}

Mechanical analysis of 16 emboli retrieved from 11 stroke patients with LVO was successfully performed, as described above. Data derived from 21 specimens were excluded because of embolus fracture at a hemostat clamp or slippage off a clamp. Under increasing load, all emboli elongated until final dissociation, demonstrating focal, multifocal, cavitated, or stress-concentrated fractures (Supplemental Material and Video 2).

VIDEO 2. Patterns of fracture resulting from tensile tests of patient emboli, including focal, multifocal, cavitated, and stress-concentrated fractures. Copyright University of Michigan. Published with permission. Click here to view.

The tensile stress-strain test and histological results for cardioembolic, atherogenic, and cryptogenic emboli are presented in Table 1 in the Supplemental Material. The data reveal the following results (mean values are presented $\pm \mathrm{SD}$ ):

- Embolus stress increases with strain.

- Emboli causing strokes are highly heterogeneous, both mechanically and histologically, from patient to patient and within the same patient (e.g., emboli 2A-E). Mechanically, emboli have $\varepsilon_{\mathrm{ut}}$ of 1.05-4.89 and $\sigma_{\mathrm{ut}}$ of 63-2396 kPa. Histologically, emboli have 0.45\%-91\% RBCs $(43 \% \pm 35 \%), 0 \%-95 \%$ fibrin $(40 \% \pm 37 \%)$, and $1.7 \%-56 \%$ platelets $(18 \% \pm 16 \%)$.

- Emboli with a high fibrin percentage (emboli 6-8) have much higher $\sigma_{\mathrm{ut}}$ compared to the other emboli (i.e., they can tolerate higher loads).

- Ten emboli (62.5\%), represented by solid lines in Supplemental Table 1A-C, had multiple fractures $\left(n_{f}>1\right)$ during the tensile test, with a maximum of $\mathrm{n}_{\mathrm{f}}=5(\mathrm{em}$ bolus 2D) and an average of $\mathrm{n}_{\mathrm{f}}=2.7$. These emboli had $\varepsilon_{\text {ut }}$ of $1.42-4.89(2.91 \pm 1.00), \sigma_{\text {ut }}$ of $63-2396 \mathrm{kPa}(799$ 
TABLE 2. Correlation analysis results of embolus composition and strength in 16 embolic specimens

\begin{tabular}{|c|c|c|c|c|c|c|}
\hline \multirow[b]{2}{*}{ Variable } & \multicolumn{2}{|c|}{ RBC (\%) } & \multicolumn{2}{|c|}{ Fibrin (\%) } & \multicolumn{2}{|c|}{ Platelet (\%) } \\
\hline & r Statistic & $p$ Value & r Statistic & $p$ Value & r Statistic & $p$ Value \\
\hline RBC (\%) & & & -0.897 & $<0.001^{*}$ & -0.108 & 0.691 \\
\hline Fibrin (\%) & -0.897 & $<0.001^{*}$ & & & -0.343 & 0.193 \\
\hline Platelet (\%) & -0.108 & 0.691 & -0.343 & 0.193 & & \\
\hline$\varepsilon_{\mathrm{ut}}$ & -0.274 & 0.304 & -0.011 & 0.969 & 0.608 & $0.012 \dagger$ \\
\hline$\sigma_{\mathrm{ut}}(\mathrm{kPa})$ & -0.565 & $0.023 \dagger$ & 0.670 & $0.005 \ddagger$ & -0.305 & 0.251 \\
\hline
\end{tabular}

$\pm 838 \mathrm{kPa}), 0.5 \%-71 \%$ RBCs $(23 \% \pm 25 \%), 0 \%-95 \%$ fibrin $(53 \% \pm 39 \%)$, and $3.5 \%-56 \%$ platelets $(24 \% \pm$ $18 \%)$.

- Six emboli $(37.5 \%)$ had a single or focal fracture $\left(\mathrm{n}_{\mathrm{f}}=\right.$ $1)$, with $\varepsilon_{\mathrm{ut}}$ of $1.14-2.54(1.64 \pm 0.54), \sigma_{\mathrm{ut}}$ of $76-161 \mathrm{kPa}$ $(111 \pm 31 \mathrm{kPa}), 15 \%-90 \%$ RBCs $(72 \% \pm 29 \%), 0 \%-$ $68 \%$ fibrin $(20 \% \pm 26 \%)$, and $1.7 \%-17 \%$ platelets $(8.1 \%$ $\pm 5.2 \%$ ).

- Based on one-way ANOVA testing, emboli with multiple fractures had higher $\varepsilon_{\mathrm{ut}}(\mathrm{p}=0.013)$. This is because the multiple local tearing of the emboli yields greater elongation before fracture. The ANOVA test also showed that emboli with multiple fractures had higher $\sigma_{u t}$, but this finding was less significant $(\mathrm{p}=0.068)$.

- For emboli obtained from LVO strokes related to carotid artery disease $(n=3), \varepsilon_{\mathrm{ut}}$ ranged from 3.05 to 4.89 and $\sigma_{\mathrm{ut}}$ ranged from 63 to $685 \mathrm{kPa}$.

- Emboli obtained from LVO strokes related to cardioembolic sources $(n=12)$ had $\varepsilon_{\mathrm{ut}}$ of 1.14-3.75 $(2.08 \pm$ $0.78)$ and higher $\sigma_{\text {ut }}$ of $76-2396 \mathrm{kPa}(601 \pm 833 \mathrm{kPa})$.

- The strongest embolus encountered was related to a left ventricular assist device with $\varepsilon_{\mathrm{ut}}$ of 3.75 and $\sigma_{\mathrm{ut}}$ of 2396 $\mathrm{kPa}$.

Table 2 shows the results of Pearson correlation analysis among embolus composition (RBC, fibrin, and platelet), $\sigma_{\mathrm{ut}}$, and $\varepsilon_{\mathrm{ut}}$ for the 16 patient emboli:

- Fibrin percentage was strongly and inversely correlated with the RBC percentage ( $\mathrm{r}=-0.897, \mathrm{p}<0.001)$.

- The $\varepsilon_{\mathrm{ut}}$ was strongly and positively correlated with the platelet percentage $(\mathrm{r}=0.608, \mathrm{p}=0.012)$. Therefore, platelet-rich emboli tended to elongate more when exposed to load.

- The $\sigma_{\text {ut }}$ is moderately and inversely correlated with the RBC percentage $(r=-0.565, \mathrm{p}=0.023)$ and strongly and positively correlated with the fibrin percentage $(\mathrm{r}=$ $0.670, p=0.005)$. Therefore, RBC-rich emboli tended to fracture at lower loads than fibrin-rich emboli.

\section{Discussion}

This study was conducted to gain further understanding of the forces driving the complex interaction between emboli and current thrombectomy devices. The main force driving embolus removal by aspiration catheters and stent retrievers was uniaxial tension. In the phantom, this force caused EAs to elongate, thin, fracture, and embolize.

For the aspiration catheters, the soft emboli will deform, enter into the catheter lumen, and be removed exclusively by intracatheter elongation and fracture. We attempted to reproduce this condition but could not draw meaningful data, as this type of embolus repeatedly fragmented on introduction in the pressurized phantom and fully migrated downstream to the collection canister. The EAs that withstood the flow and pressure of our anatomically and physiologically accurate phantoms did enter the distal-most 5-10 $\mathrm{mm}$ of the aspiration catheter at full vacuum, but they then clogged the catheter (Figs. 1A and 4A). This result is commonly observed in clinical practice and explains the need for multiple attempts and catheter unclogging. Mechanistically, it may be due to the accumulation at the catheter tip of static friction that overwhelms the vacuum power. At this point, withdrawal of the catheter exerts a tensile force on the embolus between the engagement segment and the embolus-vascular surface interface. If the embolus is stronger than the total resistance forces, it will eventually disengage from the vasculature and be removed. In this process, the embolus elongates and becomes thinner, undergoing one or more fractures and possibly fragmentation, leading it to recoil back to the obstructed artery or leading to iatrogenic embolization (Fig. 4B). This failure mechanism was recurrent in the thrombectomy procedure in our model and is also likely in clinical settings.

In a similar fashion, the current stent retriever can successfully remove an embolus from an artery when the stent integrates sufficiently with the embolus to exert a tensile force greater than the overall resistance forces. For this to happen, the embolus must be mechanically strong and homogeneous enough to resist the tensile stress and the concentrated stress near the stent tines during pulling (Fig. 4C). Even with good integration of the embolus with the stent tines, pulling of the stent will likely lead to embolus elongation and fracture in the weakest regions, followed by fracture in stronger regions and increased stress that leads to uncontrolled and multifocal embolus fragmentation. In turn, this may lead to disengagement of the embolus from the stent and fragmentation downstream (Fig. 4D). This process likely prevails where the embolus 

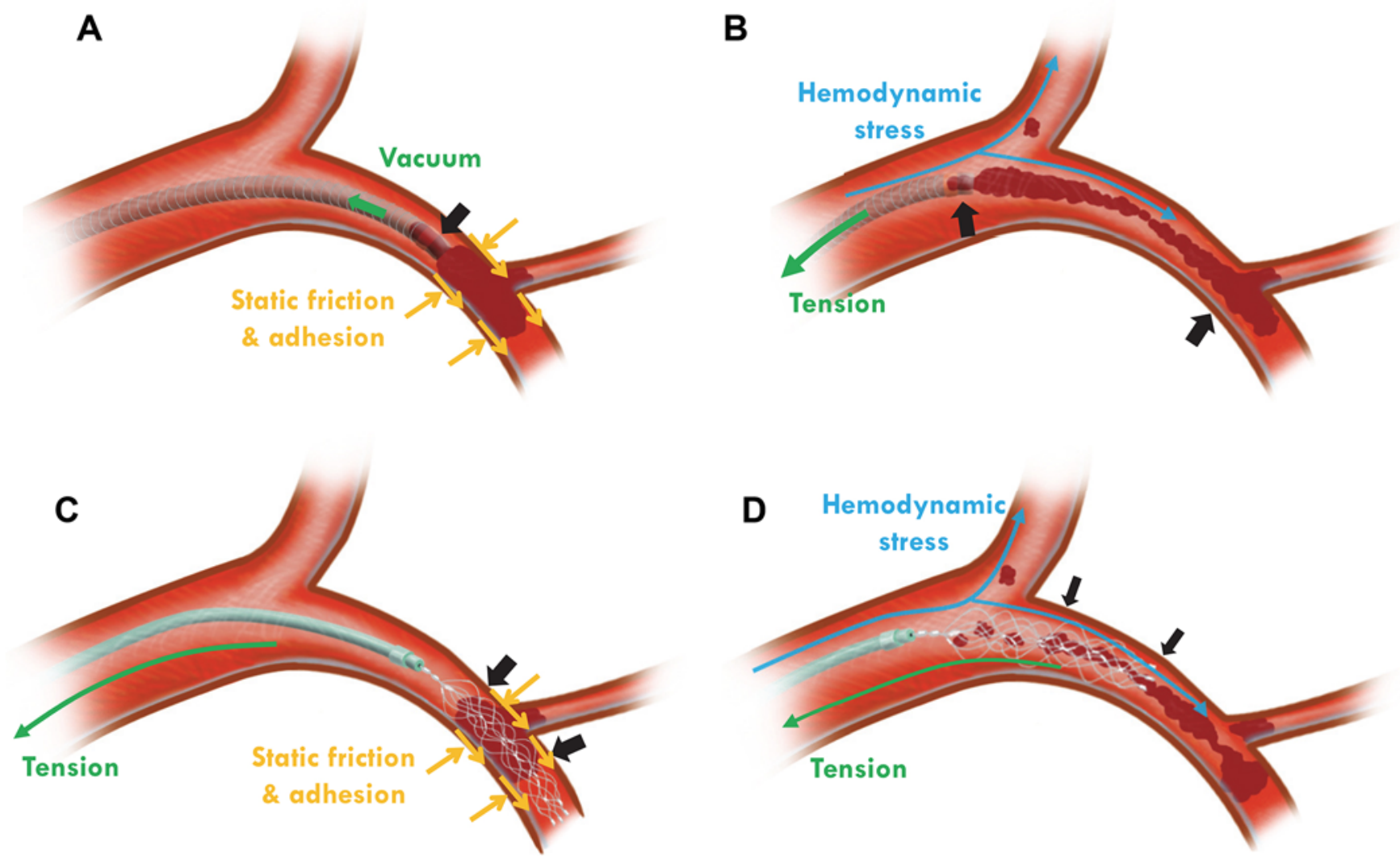

FIG. 4. Forces involved in the removal of an embolus in LVO with an aspiration catheter and stent retriever. A: In the case of the aspiration catheter, at the impaction stage, the vacuum engages the embolus (black arrow). B: Withdrawal of the catheter exerts tensile forces at the embolus between the engagement segment and the embolus-vascular surface interface (black arrows). The loaded embolus elongates, fractures, and releases fragments that embolize downstream by blood flow (blue arrows). C: In the case of the stent retriever, the tension is applied at the region of stent-emboli integration (black arrows), which generally overlaps areas of maximal resistance forces. D: Further tension leads to embolus elongation, weakening, and multifocal fragmentation, with decreased embolus integration (black arrows) concurrent with increased hemodynamic stress due to restored blood flow. Figure is available in color online only.

contacts the stent tines with large concentrated stress. The same phenomenon can be seen at branching points where subtotal stent deployment compromises embolus-device interaction, as shown in our analysis. This finding may explain the occurrence of macroembolization in the same or new vascular territories following stent thrombectomy. Iatrogenic embolizations were consistently observed during thrombectomy procedures in our model and were the main causes of incomplete recanalization. In this regard, in the experimental platform here presented, the CAPTIVE technique was more frequently associated with embolization and needed more passes for successful recanalization than the ADAPT technique. This finding may reflect structural weakening of emboli during deployment of stents through the embolus substance and concentrated stress near the stent tines during pulling, which leads to embolus fragmentation.

To date, most work dedicated to the analysis of emboli causing stroke has concentrated on histological characterization. ${ }^{2,9,13,18}$ Although valuable, histology per se is a limited proxy of mechanical strength and does not provide information related to the dynamic interaction of the em- bolus and thrombectomy device when retrieval forces are applied. Previous efforts to understand the complex mechanical behavior of emboli have utilized compression, ${ }^{4,5}$ rheometer, $8,17,21,22$ and shear wave elastography. ${ }^{7}$ These tests characterize the embolic elastic modulus and viscoelastic properties defining the deforming behavior of the embolus under mechanical load. However, these mechanical features are not the primary drivers of current thrombectomy devices, which are designed to "pull" emboli. Only two papers in the literature have described the tensile stress-strain relationships of EAs from porcine whole blood ${ }^{12}$ and bovine whole blood. ${ }^{15}$ The strengths of these EAs were about $6 \mathrm{kPa}$ and $30 \mathrm{kPa}$, respectively, about 80 times weaker than some of the samples analyzed in our cohort, raising questions about the external validity (and generalizability to clinical scenarios) of the results of thrombectomy devices tested with these EAs. Our data also show that human emboli are mechanically and structurally heterogeneous, limiting the value of tensile analysis of homogeneous EAs.

Both aspiration catheters and stent retrievers are based on the classic paradigm of "en bloc" removal of an embolus by the generation of tensile forces (Figs. 3 and 4) 
and may lead to embolization due to the pulling. The tensile forces must overcome the resistance forces (mostly static friction and adhesion of the embolus to the vascular surface plus the pressure gradient across the embolus plus material strength) by applying a load, and in this process the embolus elongates and weakens, which leads to possible fracture and iatrogenic embolization. This phenomenon is enhanced by the reduction of the embolus' diameter related to elongation, with at least partial luminal reconstitution that allows flow through the previously stagnant arterial segment, which in turn favors downstream showering of embolus fragments. In the present study, $62.5 \%$ $(10 / 16)$ of the patient-derived emboli were associated with inhomogeneous elongation, with multifocal thinning and weakening likely leading to a highly emboligenic situation in the presence of arterial flow.

In addition, we demonstrated that emboli from patients are generally highly heterogeneous, both histologically and mechanically, and therefore may elongate and fracture at different stresses during thrombectomy. Emboli were composed of $0.45 \%-91 \%$ RBCs $(43 \% \pm 35 \%), 0 \%-$ $95 \%$ fibrin $(40 \% \pm 37 \%)$, and $1.7 \%-56 \%$ platelets $(18 \% \pm$ $16 \%$, which is in line with the values found in the literature. ${ }^{2,9,13,18}$ Likewise, tensile strength varied greatly, with embolus fractures occurring at strains of 1.05-4.89 (2.41 \pm $1.04)$ and stresses of $63-2396 \mathrm{kPa}(569 \pm 695 \mathrm{kPa})$. These results reflect the highly heterogeneous and varied compositions of emboli, both among patients and within the same patient (possibly related to different embolus ages), and support a strong and significant $(\mathrm{p}<0.05)$ dependence of embolus strength on composition. These factors, along with higher resistance forces due to prolonged embolusendothelial interaction in patients, suggest that fragmentation and iatrogenic embolization may be significantly more common than observed in the simulator and are likely underdiagnosed in clinical practice.

Another finding of our study was that under tension, the patient emboli became stiffer as the strain increased, showing a strong strain-hardening property. As shown in the stress-strain curves, embolus stress increases with strain and increases faster as the strain becomes greater. A similar phenomenon has been found in tensile tests of EAs. ${ }^{12,15}$ Two possible explanations for this finding are as follows: 1) as the embolus elongates, the weaker part of the embolus fractures, leaving the stronger structural network behind to elongate, and 2) the individual fibrin fibers are material with a strain-hardening effect. ${ }^{22}$ Upon prolonged aspiration or repeated stent pulling, emboli may harden and become further compacted, making further thrombectomy attempts less likely to succeed. This is especially relevant with greater embolus length, as a larger surface area between the embolus and the vessel would yield to higher resistance forces.

We acknowledge several limitations. First, the model does not mimic the biological interaction of the endothelium with the embolus, nor does it mimic the process of water-hammer compaction by the bloodstream due to occlusion prior to thrombectomy. We speculate that in our patients, the resistance forces were significantly stronger than those generated in our model, which would necessitate higher tensile forces to remove the emboli. Second, the EAs used in the model were homogeneous in structure, and therefore the model likely underestimates the incidence of multifocal fracture and embolization. Third, the emboli tested were fragments retrieved during thrombectomy rather than the whole embolus occluding the artery. Therefore, in addition to the selection bias of testing only emboli that could actually be retrieved, it is possible that the specimens analyzed may have lacked difficultto-remove emboli and highly emboligenic material that fragmented and was lost during retrieval. Finally, in our experiments we analyzed the mechanical properties of emboli in a quasi-static condition to simulate the slow and steady withdrawal of devices that is common in the clinical setting. However, the embolus material is viscoelastic, meaning the stress on the embolus material depends on both the magnitude of elongation and the rate of elongation. Higher rates of elongation, or faster speed of device withdrawal, make the embolus behave in a stiffer manner, and this could as well affect the static friction between the embolus and suction catheter, which is a component of the pulling forces. Future studies should be conducted to analyze the interplay of variables affecting the success of thrombectomy procedures and devices, including but not limited to the speed of device withdrawal, cyclic versus continuous vacuum, length of EAs engaged in the aspiration catheter, and ideal stent deployment in relationship to embolus length and suction catheter placement.

\section{Conclusions}

Current thrombectomy devices remove emboli mostly by applying tensile forces. Emboli elongate until dissociation, which is preceded by focal fracture, multifocal fracture, cavitated fracture, or stress-concentrated fracture. Embolus strength is highly variable and significantly correlates with the embolus composition, which varies among emboli and within the same embolus. The dynamic intravascular weakening of emboli during removal with current devices under physiological flow conditions leads to a stage prone to iatrogenic embolization and the need for multiple attempts to completely recanalize arteries.

\section{Acknowledgments}

We thank neurointerventional radiology technicians at the University of Michigan for their technical assistance in obtaining emboli from thrombectomy procedures. We are also grateful to Megan Foldenauer for artwork assistance.

Funding was received from the U-M Coulter Translational Research Partnership Program and the NIH Centers for Accelerated Innovations of the NHLBI.

\section{References}

1. Blanc R, Redjem H, Ciccio G, Smajda S, Desilles JP, Orng $E$, et al: Predictors of the aspiration component success of a direct aspiration first pass technique (ADAPT) for the endovascular treatment of stroke reperfusion strategy in anterior circulation acute stroke. Stroke 48:1588-1593, 2017

2. Boeckh-Behrens T, Schubert M, Förschler A, Prothmann S, Kreiser K, Zimmer C, et al: The impact of histological clot composition in embolic stroke. Clin Neuroradiol 26:189197, 2016

3. Chartrain AG, Awad AJ, Mascitelli JR, Shoirah H, Oxley TJ, 
Feng R, et al: Novel and emerging technologies for endovascular thrombectomy. Neurosurg Focus 42(4):E12, 2017

4. Chueh JY, Wakhloo AK, Hendricks GH, Silva CF, Weaver JP, Gounis MJ: Mechanical characterization of thromboemboli in acute ischemic stroke and laboratory embolus analogs. AJNR Am J Neuroradiol 32:1237-1244, 2011

5. Dempfle CE, Kälsch T, Elmas E, Suvajac N, Lücke T, Münch E, et al: Impact of fibrinogen concentration in severely ill patients on mechanical properties of whole blood clots. Blood Coagul Fibrinolysis 19:765-770, 2008

6. Gunning GM, McArdle K, Mirza M, Duffy S, Gilvarry M, Brouwer PA: Clot friction variation with fibrin content; implications for resistance to thrombectomy. J Neurointerv Surg 10:34-38, 2018

7. Huang CC, Chen PY, Shih CC: Estimating the viscoelastic modulus of a thrombus using an ultrasonic shear-wave approach. Med Phys 40:042901, 2013

8. Kim OV, Litvinov RI, Weisel JW, Alber MS: Structural basis for the nonlinear mechanics of fibrin networks under compression. Biomaterials 35:6739-6749, 2014

9. Kim SK, Yoon W, Kim TS, Kim HS, Heo TW, Park MS: Histologic analysis of retrieved clots in acute ischemic stroke: correlation with stroke etiology and gradient-echo MRI.

AJNR Am J Neuroradiol 36:1756-1762, 2015

10. Kleine JF, Wunderlich S, Zimmer C, Kaesmacher J: Time to redefine success? TICI 3 versus TICI $2 b$ recanalization in middle cerebral artery occlusion treated with thrombectomy. J Neurointerv Surg 9:117-121, 2017

11. Klinger-Gratz PP, Schroth G, Gralla J, Jung S, Weisstanner C, Verma RK, et al: Protected stent retriever thrombectomy prevents iatrogenic emboli in new vascular territories. Neuroradiology 57:1045-1054, 2015

12. Krasokha N, Theisen W, Reese S, Mordasini P, Brekenfeld C, Gralla J, et al: Mechanical properties of blood clots - A new test method. Materialwiss Werkstofftech 41:1019-1024, 2010

13. Liebeskind DS, Sanossian N, Yong WH, Starkman S, Tsang MP, Moya AL, et al: CT and MRI early vessel signs reflect clot composition in acute stroke. Stroke 42:1237-1243, 2011

14. Liu Y, Zheng Y, Li AD, Liu Y, Savastano LE, Shih AJ: Cutting of blood clots-experiment and smooth particle Galerkin modelling. CIRP Annals 68:97-100, 2019

15. Malone F, McCarthy E, Delassus P, Fahy P, Kennedy J, Fagan AJ, et al: The mechanical characterisation of bovine embolus analogues under various loading conditions. Cardiovasc Eng Technol 9:489-502, 2018

16. McTaggart RA, Tung EL, Yaghi S, Cutting SM, Hemendinger $\mathrm{M}$, Gale HI, et al: Continuous aspiration prior to intracranial vascular embolectomy (CAPTIVE): a technique which improves outcomes. J Neurointerv Surg 9:1154-1159, 2017

17. Merritt W, Holter AM, Beahm S, Gonzalez C, Becker TA, Tabor A, et al: Quantifying the mechanical and histological properties of thrombus analog made from human blood for the creation of synthetic thrombus for thrombectomy device testing. J Neurointerv Surg 10:1168-1173, 2018

18. Niesten JM, van der Schaaf IC, van Dam L, Vink A, Vos JA, Schonewille WJ, et al: Histopathologic composition of cerebral thrombi of acute stroke patients is correlated with stroke subtype and thrombus attenuation. PLoS One 9:e88882, 2014
19. Palaniswami M, Yan B: Mechanical thrombectomy is now the gold standard for acute ischemic stroke: Implications for routine clinical practice. Intervent Neurol 4:18-29, 2015

20. Turk AS, Spiotta A, Frei D, Mocco J, Baxter B, Fiorella D, et al: Initial clinical experience with the ADAPT technique: a direct aspiration first pass technique for stroke thrombectomy. J Neurointerv Surg 6:231-237, 2014

21. van Kempen TH, Donders WP, van de Vosse FN, Peters GW: A constitutive model for developing blood clots with various compositions and their nonlinear viscoelastic behavior. Biomech Model Mechanobiol 15:279-291, 2016

22. van Kempen TH, Peters GW, van de Vosse FN: A constitutive model for the time-dependent, nonlinear stress response of fibrin networks. Biomech Model Mechanobiol 14:9951006, 2015

23. Yoo AJ, Andersson T: Thrombectomy in acute ischemic stroke: challenges to procedural success. J Stroke 19:121130,2017

\section{Disclosures}

Dr. Savastano reports an ownership stake in Endovascular Engineering, for which he was a cofounder and which is developing a new technology for stroke and therefore could potentially have indirect conflict of interest with stent and suction catheters.

\section{Author Contributions}

Conception and design: Savastano, Liu, Zheng, Shih. Acquisition of data: Liu, Reddy, Gebrezgiabhier, Davis, Cockrum. Analysis and interpretation of data: Liu. Drafting the article: Savastano, Liu. Critically revising the article: Savastano, Liu, Pandey, Shih. Reviewed submitted version of manuscript: all authors. Approved the final version of the manuscript on behalf of all authors: Savastano. Statistical analysis: Liu. Administrative/technical/ material support: Savastano, Gemmete, Chaudhary, Griauzde, Pandey. Study supervision: Savastano, Pandey, Shih.

\section{Supplemental Information \\ Videos}

Video 1. https://vimeo.com/380005321.

Video 2. https://vimeo.com/380005351.

\section{Online-Only Content}

Supplemental material is available with the online version of the article.

Supplemental Material. https://thejns.org/doi/suppl/10.3171/ 2019.12.JNS192187.

\section{Correspondence}

Luis E. Savastano: Mayo Clinic, Rochester, MN. savastano.luis@ mayo.edu. 